\title{
SELF-FOCUSING AND SELF-TRAPPING OF SPHERCIAL BEAMS IN A NONLINEAR MEDIUM
}

\author{
KIN-CHUNG NG
}

\begin{abstract}
Self-focusing and self-trapping of optical beams are studied by obtaining the asymptotic solution of the nonlinear reduced wave equation

$$
\nabla^{2} u+k^{2} n^{2}\left(|u|^{2}\right) u=0
$$

in three dimensional space where the incident waves are assumed to be spherical waves. In order to discuss diffraction effects and self-focusing effects of the beam, the concepts of diffraction length and focal length are introduced. It is shown that diffraction effects and self-focusing effects occur in different regions. This means that diffraction cannot, in general, influence self-focusing. In the special case where diffraction effects and self-focusing effects are balanced, as self-trapped beam is shown to exist.
\end{abstract}

\section{Introduction}

In the theory of nonlinear optics (see [1]), one is led to consider a nonlinear reduced wave equation

$$
\nabla^{2} u+k^{2} n^{2}\left(|u|^{2}\right) u=0
$$

where $k$ is the wave number and $n=n\left(|u|^{2}\right)$ is a function of intensity of the field and is called the index of refraction.

We consider the case when the optical beam propagates in a quadratic index media so that $n^{2}\left(|u|^{2}\right)$ can be written as

$$
n^{2}\left(|u|^{2}\right)=n_{0}^{2}+n_{1}|u|^{2}, \quad n_{1}>0
$$

Received June 30, 1993; revised November 11, 1993.

1991 Mathematics Subject Classification. 35A35, 35C20, 35Q60.

Key words and phrases. Self-focusing, self-trapping, spherical beams, nonlinear reduced wave equation. 
where $n_{0}$ and $n_{1}$ are constants.

With $|u|=O\left(k^{-d}\right), d>0$, and following the discussion of this equation in two dimensional space (see [2]), we write

$$
u=\frac{1}{k^{d}} a e^{i k \phi} .
$$

Substituting (3) into (1) and equating real and imaginary parts, we obtain

and.

$$
\left\{\left[(\nabla \phi)^{2}-n_{0}^{2}\right] a-\frac{1}{k^{2 d}} n_{1} a^{3}-\frac{1}{k^{4 d}} n_{2} a^{5}+\cdots\right\}-\frac{1}{k^{2}} \nabla^{2} a=0
$$

$$
2 \nabla \phi \cdot \nabla a+a \nabla^{2} \phi=0 .
$$

The most interesting cases for $d$ are $d=1$ and $d=\frac{1}{2}$. If $d=1$ then $|u|=O\left(k^{-1}\right)$ and we have a self-trapped beam which is the analog of the solitary wave in unsteady problems. We discuss that case in section 3. In the first part of this paper, we consider the case when $d=\frac{1}{2}$. Then we expand $\phi$ and $a$ as

$$
\left\{\begin{array}{l}
\phi=\sum_{j=0}^{\infty} \phi_{j} k^{-j} \\
a=\sum_{j=0}^{\infty} a_{j} k^{-j} .
\end{array}\right.
$$

On substituting (5) and (6) into (4) and equating coefficcents of like powers of $k^{-1}$, we obtain

and

$$
\begin{aligned}
& \left(\nabla \phi_{0}\right)^{2}=n_{0}^{2} \\
& 2 \nabla \phi_{0} \cdot \nabla a_{0}+a_{0} \nabla^{2} \phi_{0}=0 \\
& 2 \nabla \phi_{0} \cdot \nabla \phi_{1}=n_{1} a_{0}^{2} \\
& 2 \nabla \phi_{0} \cdot \nabla a_{1}+a_{1} \nabla^{2} \phi_{0}=-2 \nabla \phi_{1} \cdot \nabla a_{0}-a_{0} \nabla^{2} \phi_{1} \\
& 2 \nabla \phi_{0} \cdot \nabla \phi_{2}=n_{2} a_{0}^{4}+3 n_{1} a_{0} a_{1}+\frac{1}{a_{0}} \nabla^{2} a_{0}-\frac{2 a_{1}}{a_{0}} \nabla \phi_{0} \cdot \nabla \phi_{1}-\left(\nabla \phi_{1}\right)^{2}
\end{aligned}
$$

$$
2 \nabla \phi_{0} \cdot \nabla a_{2}+a_{2} \nabla^{2} \phi_{0}=-2 \nabla \phi_{2} \cdot \nabla a_{0}-a_{0} \nabla^{2} \phi_{2}-2 \nabla \phi_{1} \cdot \nabla a_{1}-a_{1} \nabla^{2} \phi_{1} .
$$

Here we will not try to obtain the general solutions of these equations. (For the plane wave case in two dimensional space, a detailed discussion of the solution can be found in [2].) We will discuss the behavior of a spherical beam $u(r, z)$ propagating in a nonlinear medium for large distances.

\section{Self-focusing of a spherical beam}

We now consider the case where the incoming wave is spherical with an amplitude which has axial symmetry with respect to the $z$-axis. Introducing cylindrical coordinates, the phase term $\phi_{0}$ of the incoming wave can be written as

$$
\phi_{0}=\phi_{0}(r, z)=\sqrt{(z+R)^{2}+r^{2}}
$$


where we have assumed that the focal point is located at $z=-R$ and $r=0$ and that the unperturbed index of refraction $n_{0}$ equals 1 . Then

$$
\left(\nabla \phi_{0}\right)^{2}=\left(\frac{\partial \phi_{0}}{\partial r}\right)^{2}+\left(\frac{\partial \phi_{2}}{\partial z}\right)^{2}=\frac{r^{2}}{\phi_{0}^{2}}+\frac{(z+R)^{2}}{\phi_{0}^{2}}=1 .
$$

Near the axis of the beam where $r \approx 0$ we can approximate $\phi_{0}$ as

$$
\phi_{0} \approx z+R+\frac{r^{2}}{2(z+R)}
$$

Because of the assumed axial symmetry we write $a_{0}=a_{0}(r, z)$ and obtain from (8)

$$
\frac{2}{\phi_{0}}\left[(z+R) \frac{\partial a_{0}}{\partial z}+r \frac{\partial a_{0}}{\partial r}+a_{0}\right]=0
$$

The general solution of (16) with the arbitrary function $f\left[\frac{r}{1+\frac{z}{R}}\right]$ determined from the form of the incident wave at $z=0$ is

$$
a_{0}(r, z)=R f\left[\frac{r}{1+\frac{z}{R}}\right] / \sqrt{(z+R)^{2}+r^{2}} .
$$

Thus $a_{0}=R f(r) / \sqrt{R^{2}+r^{2}}$ at $z=0$, and hence $a_{0} \rightarrow f(r)$ as $R \rightarrow \infty$. Then our problem reduces to the plane wave case in three dimensional space. By setting $f\left[\frac{r}{1+\frac{z}{R}}\right]=$ $E \exp \left[-\frac{r^{2}}{\alpha^{2}\left(1+\frac{z}{R}\right)^{2}}\right]$ and $x=0$, we readily see that our result agrees with the known result for the plane wave case in two dimensional space (see [2]). In order that the incident wave represents a beam we assume that $f(r) \rightarrow 0$ as $r \rightarrow \infty$ and that $f^{\prime}(0)=0$ with $f^{\prime \prime}(0)<0$ so that the amplitude has a maximum at $r=0$. From (9) we have

$$
(z+R) \frac{\partial \phi_{1}}{\partial z}+r \frac{\partial \phi_{1}}{\partial r}=\frac{1}{2} n_{1} R^{2} f^{2} / \sqrt{(z+R)^{2}+r^{2}} .
$$

Since we require that $\phi_{1}(r, 0)=0$ so that the incident wavefront is spherical, we have as a solution of (18)

$$
\phi_{1}(r, z)=\frac{1}{2} n_{1} f^{2} \frac{R z}{\sqrt{(z+R)^{2}+r^{2}}} .
$$

As $R \rightarrow \infty$ the above result tends to $\phi_{1}=\frac{1}{2} n_{1} f^{2}(r) z$ which gives the result for plane wave incidence. For small $r$ that is near the axis of the beam, (19) can be expanded as

$$
\phi_{1}=\frac{1}{2} n_{1} f^{2}\left[\frac{z}{1+\frac{z}{R}}-\frac{1}{2} \frac{r^{2} z}{\left(1+\frac{z}{R}\right)(z+R)}+\cdots\right] .
$$

From (10) the equation for $a_{1}$ becomes

$$
\frac{2}{\phi_{0}}\left[(z+R) \frac{\partial a_{1}}{\partial z}+r \frac{\partial a_{1}}{\partial r}+a_{1}\right]=-2 \nabla \phi_{1} \cdot \nabla a_{0}-a_{0} \nabla^{2} \phi_{1} .
$$


It is possible to solve (21) with $a_{1}(r, 0)=0$ exactly, but the result is fairly complicated. Our main interest is to determine the self-focusing length for the beam, which results from the secular behavior of the asymptotic expansion and is determined by the behavior of $a_{1}$ for large $z$ and small $r$. The main contribution to the right side of (21) for large $z$ and small $r$ can be shown to come from the terms $\frac{1}{r} a_{0} \frac{\partial \phi_{1}}{\partial r}$ and $a_{0} \frac{\partial^{2} \phi_{1}}{\partial r^{2}}$. Since

$$
\left.\left\{f^{\prime} \frac{r}{1+\frac{z}{R}}\right]-f^{\prime}(0)\right\} / \frac{r}{1+\frac{z}{R}} \approx f^{\prime \prime}(0)
$$

as $r$ is small, and $f^{\prime}(0)=0$, we have

$$
\frac{f^{\prime}}{r}=\frac{f^{\prime}}{\frac{r}{1+\frac{z}{R}}} \cdot \frac{1}{1+\frac{z}{R}} \approx f^{\prime \prime}(0) \frac{R}{z+R} .
$$

We find that (21) is approximated as

$$
\frac{2}{\phi_{0}}\left[(z+R) \frac{\partial a_{1}}{\partial z}+r \frac{\partial a_{1}}{\partial r}+a_{1}\right]=-\frac{2 R E B z}{(z+R)^{4}}
$$

where $E=f(0)$ and $B=n_{1} E f^{\prime \prime}(0) R^{3}-\frac{1}{2} n_{1} E^{2} R$. Equation (24) can be written as

$$
(z+R) \frac{\partial a_{1}}{\partial z}+r \frac{\partial a_{1}}{\partial r}+a_{1}=-\frac{E B R}{(z+R)^{2}}+\frac{E B R^{2}}{(z+R)^{3}} .
$$

Solving (25) with $a_{1}(r, 0)=0$, we obtain

$$
a_{1}(r, z)=-\frac{E B z^{2}}{2(z+R)^{3}} .
$$

For small $r$ and large $z, a_{0}(r, z)$ can be written as

$$
a_{0}(r, z) \approx \frac{R E}{z+R} .
$$

In order to determine the value of $z$ at which the asymptotic series becomes disordered, we set $\frac{a_{1}}{R} \approx a_{0}$. From (26) and (27) we conclude that

$$
\frac{-B z^{2}}{k 2(z+R)^{2}} \approx R
$$

(28) can be written as

We note that if

$$
\frac{1}{z} \approx \pm \sqrt{\frac{n_{1} E^{2}}{4 k R^{2}}-\frac{n_{1} E F^{\prime \prime}(0)}{2 k}}-\frac{1}{R} .
$$

$$
f(\mathrm{X})=E e^{-\mathrm{X}^{2} / \alpha^{2}}
$$


then (29) becomes

$$
\frac{1}{z} \approx \pm \sqrt{\frac{n_{1} E^{2}}{4 k R^{2}}+\frac{n_{1} E^{2}}{\alpha^{2} k}}-\frac{1}{R} .
$$

For finite $R$ there are two possible focal points as was found by Akhmanov et al. [3], but the term $n_{1} E^{2} / 4 k R^{2}$ does not appear in their result. If we assume that the beam is thin in the sense that $0<\alpha \ll 1$, then we find that $\frac{1}{\alpha^{2}} \gg \frac{1}{R^{2}}$, and hence the focal length (s) determined from (29) or (31) reduces to the results of plane wave case, i.e., we have

$$
\frac{1}{z_{f}} \approx \pm \sqrt{\frac{n_{1} E^{2}}{\alpha^{2} k}}
$$

To determine the diffraction length for the spherical beam, we have to know $a_{2}(r, z)$. In the absence of nonlinear effects, (11) becomes

$$
2 \nabla \phi_{0} \cdot \nabla \phi_{2}=\frac{1}{a_{0}} \nabla^{2} a_{0}
$$

If we set $f=E \exp \left[-\frac{r^{2}}{\alpha^{2}\left(1+\frac{z}{R}\right)^{2}}\right]$, substitute (13) and (17) into (33), and keep only the most significant terms, we obtain

$$
(z+R) \frac{\partial \phi_{2}}{\partial z}+r \frac{\partial \phi_{2}}{\partial r}=\frac{2 R^{4} r^{2}}{\alpha^{4}(z+R)^{3}}-\frac{2 R^{2}}{\alpha^{2}(z+R)}
$$

Solving (34) with condition $\phi_{2}(r, 0)=0$, we have

$$
\phi_{2}(r, z)=\frac{2 R^{3} r^{2} z}{\alpha^{4}(z+R)^{3}}-\frac{2 R z}{\alpha^{2}(z+R)} .
$$

In the absence of nonlinear effects, (12) becomes

$$
2 \nabla \phi_{0} \cdot \nabla a_{2}+a_{2} \nabla^{2} \phi_{0}=-2 \nabla \phi_{2} \cdot \nabla a_{0}-a_{0} \nabla^{2} \phi_{2}
$$

Substitute (13), (17) and (35) into (36), and keep the most significant terms, we have

$$
(z+R) \frac{\partial a_{2}}{\partial z}+r \frac{\partial a_{2}}{\partial r}+a_{2}=-\frac{4 R^{4} E z}{\alpha^{4}(z+R)^{3}} .
$$

Solving (37) with condition $a_{2}(r, 0)=0$, we obtain

$$
\begin{aligned}
a_{2}(r, z) & \approx-\frac{4 R^{4} E}{\alpha^{4}}\left[\frac{R}{2(z+R)^{3}}-\frac{1}{(z+R)^{2}}+\frac{1}{2(z+R) R}\right] \\
& =-\frac{2 R^{3} E z^{2}}{\alpha^{4}(z+R)^{3}} .
\end{aligned}
$$


For a linear medium, the asymptotic expansion of the amplitude $a$ can be written as

$$
\begin{aligned}
a(r, z) & \approx a_{0}+\frac{1}{k} a_{1}+\frac{1}{k^{2}} a_{2} \\
& \approx \frac{R E}{z+R}-\frac{1}{k^{2}} \frac{2 R^{3} E z^{2}}{\alpha^{4}(z+R)^{3}} .
\end{aligned}
$$

Thus the secular effects occur when

$$
\frac{R E}{z+R} \approx \frac{1}{k^{2}} \frac{2 R^{3} E z^{2}}{\alpha^{4}(z+R)^{3}}
$$

which implies

$$
z \approx \frac{k \alpha^{2}}{\sqrt{2}}\left(1+\frac{z}{R}\right)
$$

When $R \rightarrow \infty,(41)$ reduces to the plane wave case. The diffraction length is given by

$$
z_{d}=\frac{k \alpha^{2}}{\sqrt{2}}
$$

From (32) and (42), we conclude that self-focusing effects and diffraction effects occur in different regions. In general, diffraction cannot influence self-focusing. But in some special situations diffraction and self-focusing might happen in the same region; then they do influence each other. To see this, we express the amplitude $a(r, z)$ as a combination of $a_{0}, a_{1}$ and $a_{2}$, where $a_{0}$ and $a_{2}$ are obtained for the linear case and $a_{1}$ is from a nonlinear medium. Thus we have the following asymptotic expansion

$$
\begin{aligned}
a(r, z) & \approx a_{0}+\frac{1}{k} a_{1}+\frac{1}{k^{2}} a_{2} \\
& \approx \frac{R E}{z+R}+\frac{1}{k} \frac{n_{1} E^{3} R^{3} z^{2}}{\alpha^{2}(z+R)^{3}}-\frac{1}{k^{2}} \frac{2 R^{3} E z^{2}}{\alpha^{4}(z+R)^{3}} .
\end{aligned}
$$

When

$$
\frac{R E}{z+R} \approx\left[\frac{1}{k} \frac{n_{1} E^{3} R^{3}}{\alpha^{2}(z+R)^{3}}-\frac{1}{k^{2}} \frac{2 R^{3} E}{\alpha^{4}(z+R)^{3}}\right] z^{2},
$$

the asymptotic expansion of $a(r, z)$ becomes disordered, so we have

$$
\frac{1}{R}+\frac{1}{z} \approx \pm \sqrt{\frac{n_{1} E^{2}}{k \alpha^{2}}-\frac{2}{k^{2} \alpha^{4}}} .
$$

According to (32) and (42), (45) can be written as

$$
\frac{1}{z_{s}} \approx \pm \sqrt{\frac{1}{z_{f}^{2}}-\frac{1}{z_{d^{2}}}}-\frac{1}{R}
$$


where $z_{s}$ is called the self-focusing length of the beam in the spherical wave case. If $z_{f}^{2}>z_{d^{2}}$ or $E^{2}<\frac{2}{\alpha^{2} n_{1} k}$, self-focusing is impossible because $z_{s}$ becomes a complex number. If $z_{f}^{2}<z_{d}^{2}$, then we may have two focal points when $0<\sqrt{\frac{1}{z_{f}^{2}}-\frac{1}{z_{d^{2}}}}<-\frac{1}{R}$, and only one focal point when $\sqrt{\frac{1}{z_{f}^{2}}-\frac{1}{z_{d^{2}}}}>-\frac{1}{R}>0$. We will discuss the situation when $z_{f}^{2}=z_{d}^{2}$ or $E^{2}=\frac{2}{\alpha^{2} n_{1} k}$ in the next section.

\section{Self-trapping of a spherical beam}

From the previous results, the asymptotic expansion of the phase $\phi(r, z)$ is given by

$$
\phi(r, z) \approx \phi_{0}+\frac{1}{k} \phi_{1}+\frac{1}{k^{2}} \phi_{2} \text {. }
$$

If we use (15), (35) and (19), with $f^{2}=E^{2} \exp \left[\frac{-2 r^{2}}{\alpha^{2}\left(1+\frac{z}{R}\right)^{2}}\right] \approx E^{2}\left[1-\frac{2 r^{2} R^{2}}{\alpha^{2}(z+R)^{2}}\right]$, (47) becomes

$$
\begin{aligned}
\phi(r, z) \approx & (z+R)+\frac{r^{2}}{2(z+R)}+\frac{1}{k}\left[\frac{1}{2} \frac{n_{1} E^{2} R z}{z+R}-\frac{n_{1} E^{2} R^{3} r^{2} z}{\alpha^{2}(z+R)^{3}}\right] \\
& +\frac{1}{k^{2}}\left[\frac{2 R^{3} r^{2} z}{\alpha^{4}(z+R)^{3}}-\frac{2 R z}{\alpha^{2}(z+R)}\right] .
\end{aligned}
$$

With $E^{2}=\frac{2}{\alpha^{2} n_{1} k},(48)$ can be simplified as

$$
\phi(r, z) \approx(z+R)+\frac{r^{2}}{2(z+R)}-\frac{1}{k^{2}}\left[\frac{z R}{\alpha^{2}(z+R)}\right]
$$

From (41) we know that $\frac{z R}{\alpha^{2}(z+R)}=O(k)$ in the diffraction region. We now present a boundary layer analysis of the field in the region where diffraction effects become important. We set

$$
\phi(r, z)=(z+R)+\frac{r^{2}}{2(z+R)}+\frac{1}{k} \hat{\phi}
$$

where $\hat{\phi}=O(1)$. According to (39) we have

$$
a(r, z) \approx \frac{R E}{z+R} \approx \frac{R}{(z+R)} \frac{\sqrt{2}}{\alpha \sqrt{n}_{1} \sqrt{k}}=O\left(\frac{1}{\sqrt{k}}\right) .
$$

We also set

$$
a(r, z)=\frac{1}{\sqrt{k}} \hat{a}
$$

where $\hat{a}=O(1)$. Substituting (50) and (52) into (4) and (5), with $d=\frac{1}{2}$, we obtain

$$
\begin{gathered}
{\left[\left(1-\frac{r^{2}}{2(z+R)^{2}}+\frac{1}{k} \frac{\partial \hat{\phi}}{\partial z}\right)^{2}+\left(\frac{r}{z+R}+\frac{1}{k} \frac{\partial \hat{\phi}}{\partial r}\right)^{2}-1\right]-\frac{1}{k} n_{1} a^{2}+\cdots} \\
-\frac{1}{k^{2}} \frac{1}{a} \frac{\partial^{2} a}{\partial z^{2}}-\frac{1}{k^{2}} \frac{1}{a} \frac{1}{r} \frac{\partial}{\partial r}\left(r \frac{\partial a}{\partial r}\right)=0
\end{gathered}
$$

and 


$$
\begin{aligned}
2(1- & \left.\frac{r^{2}}{2(z+R)^{2}}+\frac{1}{k} \frac{\partial \hat{\phi}}{\partial z}\right) \frac{\partial a}{\partial z}+2\left(\frac{r}{z+R}+\frac{1}{k} \frac{\partial \hat{\phi}}{\partial r}\right) \frac{\partial a}{\partial r}+\frac{a r^{2}}{(z+R)^{3}} \\
& +\frac{a}{k} \frac{\partial^{2} \hat{\phi}}{\partial z^{2}}+\frac{2 a}{z+R}+\frac{a}{k} \frac{1}{r} \frac{\partial}{\partial r}\left(r \frac{\partial \hat{\phi}}{\partial r}\right)=0
\end{aligned}
$$

To simplify the above equations, we set

$$
z=k \xi ; \quad R=k R_{0}
$$

Then $\frac{\partial}{\partial z}=\frac{1}{k} \frac{\partial}{\partial \xi}$ so that (53) and (54) become respectively

$$
\frac{1}{k^{2}}\left[2 \frac{\partial \hat{\phi}}{\partial \xi}+\frac{2 R}{\xi+R_{0}} \frac{\partial \hat{\phi}}{\partial r}+\left(\frac{\partial \hat{\phi}}{\partial r}\right)^{2}-n_{1} \hat{a}^{2}-\frac{1}{\hat{a}} \frac{1}{r} \frac{\partial}{\partial r}\left(r \frac{\partial \hat{a}}{\partial r}\right)\right]+O\left(k^{-4}\right)=0
$$

and

$$
\frac{1}{k^{\frac{3}{2}}}\left[2 \frac{\partial \hat{a}}{\partial \xi}+\frac{2 r}{\xi+R_{0}} \frac{\partial \hat{a}}{\partial r}+2 \frac{\partial \hat{\phi}}{\partial r} \frac{\partial \hat{a}}{\partial r}+\frac{\hat{a}}{r} \frac{\partial}{\partial r}\left(r \frac{\partial \hat{\phi}}{\partial r}\right)+\frac{2 \hat{a}}{\xi+R_{0}}\right]+O\left(k^{-\frac{7}{3}}\right)=0
$$

The leading terms in the expansions of $\hat{\phi}$ and $\hat{a}$ which we denote by $\hat{\phi}_{0}$ and $\hat{a}_{0}$ satisfy

and

$$
2 \frac{\partial \hat{\phi}_{0}}{\partial \xi}+\frac{2 r}{\xi+R_{0}} \frac{\partial \hat{\phi}_{0}}{\partial r}+\left(\frac{\partial \hat{\phi}_{0}}{\partial r}\right)^{2}-\frac{1}{\hat{a}_{0}} \frac{1}{r} \frac{\partial}{\partial r}\left(r \frac{\partial \hat{a}_{0}}{\partial r}\right)-n_{1} \hat{a}_{0}^{2}=0
$$

$$
2 \frac{\partial \hat{a}_{0}}{\partial \xi}+\frac{2 r}{\xi+R_{0}} \frac{\partial \hat{s}_{0}}{\partial r}+2 \frac{\partial \hat{\phi}_{0}}{\partial r} \frac{\partial \hat{a}_{0}}{\partial r}+\frac{\hat{a}_{0}}{r} \frac{\partial}{\partial r}\left(r \frac{\partial \hat{\phi}_{0}}{\partial r}\right)+\frac{2 \hat{a}_{0}}{\xi+R_{0}}=0 .
$$

Let $\hat{\phi}_{0}=\lambda \frac{R_{0}^{2}}{\xi+R_{0}}$ where $\lambda$ is an arbitrary constant and substitute it into (59). We obtain

$$
\left(\xi+R_{0}\right) \frac{\partial \hat{a}_{0}}{\partial \xi}+r \frac{\partial \hat{a}_{0}}{\partial r}+\hat{a}_{0}=0
$$

Solving (60), we obtain

$$
\hat{a}_{0}(r, z)=\frac{R_{0}}{\xi+R_{0}} \hat{F}\left[\frac{R_{0} r}{\xi+R_{0}}\right]
$$

where $\hat{F}$ is an arbitrary function we need to determine. Substituting $\hat{\phi}_{0}$ and $\hat{a}_{0}$ into (58), and letting $t=\frac{R_{0} r}{\xi+R_{0}}$, we have

$$
\hat{F}^{\prime \prime}(t)+\frac{1}{t} \hat{F}^{\prime}(t)-2 \lambda \hat{F}(t)+n_{1} \hat{F}^{3}(t)=0
$$

The approximate solution of equation (62) is known (see [4]) and is given by

$$
\hat{F}(t)=\sqrt{\frac{2 \lambda}{n_{1}}}\left\{0.85 \exp \left[-0.25(2 \lambda) t^{2}\right]+1.32 \exp \left[-1.18(2 \lambda) t^{2}\right]\right\} \text {. }
$$


From (52), (55), (61) and (63), we have

$$
\begin{aligned}
a(r, z)=\frac{1}{\sqrt{k}} & \left(\frac{R}{z+R}\right) \sqrt{\frac{2 \lambda}{n_{1}}}\left\{0.85 \exp \left[-0.50 \lambda \frac{R^{2} r^{2}}{(z+R)^{2}}\right]\right. \\
& \left.+1.32 \exp \left[-2.36 \lambda \frac{R^{2} r^{2}}{(z+R)^{2}}\right]\right\} .
\end{aligned}
$$

Thus, using (3), (50), (64) and $\hat{\phi}_{0}=\lambda \frac{R_{0}^{2}}{\xi+R_{0}}$, the field $u(r, z)$ can be written as

$$
\begin{aligned}
u(r, z)= & \frac{1}{k}\left(\frac{R}{z+R}\right) \sqrt{\frac{2 \lambda}{n_{1}}}\left\{0.85 \exp \left[-0.50 \lambda \frac{R^{2} r^{2}}{(z+R)^{2}}\right]\right. \\
& \left.+1.32 \exp \left[-2.36 \lambda \frac{R^{2} r^{2}}{(z+R)^{2}}\right]\right\} \exp \left\{i k\left[(z+R)+\frac{r^{2}}{2(z+R)}+\frac{\lambda}{k^{2}} \frac{R^{2}}{z+R}\right]\right\}
\end{aligned}
$$

According to Akhmanov et al., a field with spherical phase term $\phi(r, z)=(z+R)+\frac{r^{2}}{2(z+R)}$ and amplitude term $a^{2}=\frac{E^{2}}{\left(1+\frac{z}{R}\right)} F\left[\frac{r}{\alpha\left(1+\frac{z}{R}\right)}\right]$, where $F$ determines the field, is called a spherical beam. For example, if $F(z)=e^{-z^{2}}$, it is called a Gaussian beam. Thus, (65) is obviously a beam with spherical wavefront propagating in a nonlinear medium. Because the diffraction effects and self-focusing effects are balanced, there will be no spreading and focusing under this situation. This means that the shape of the beam will not change as it propagates over a long distance. The solution (65) may be referred to as a self-trapped spherical beam.

\section{References}

[1] P. W. Milonni and J. H. Eberly, Lasers, Wiley-Interscience, New York, 1991.

[2] E. Zauderer, Partial Differential Equations of Applied Mathematics, Wiley-Interscience, New York, 1989.

[3] S. A. Akhmanov, A. P. Sukhoruhov, and R. V. Khoklov, Self-focusing and self-trapping of intense light beams in a nonlinear medium, Soviet phys, Uspekhi, 10, 1968, 609-636.

[4] G. B. Whitham, Linear and Nonlinear Waves, Interscience, New York, 1974.

Department of Mathematics, Tamkang University, Tamsui, Taipei, R.O.C. 